\title{
Temporal Dynamics of Vegetation NDVI and its Response to Drought in Songnen Grassland
}

\author{
Qiyun Ma ${ }^{1}$, Jiquan Zhang ${ }^{1 *}$, Siqing Tong ${ }^{1}$, Zhenhua Dong ${ }^{1}$, Xuehai Nie $^{2}$, Yanan Chen ${ }^{1}$ \\ ${ }^{1}$ Institute of Natural Disaster Research, School of Environment, Northeast Normal University, Changchun 130024, \\ China \\ ${ }^{2}$ College of Resources and Environment, Northeast Agricultural University, Harbin 150030, China
}

\section{松嫩草地 NDVI 时间变化特征及其对干旱的响应关系 研究 \\ 马齐云 ${ }^{1}$, 张继权 ${ }^{1 *}$, 佟斯琴 ${ }^{1}$, 董振华 ${ }^{1}$, 聂学海 ${ }^{2}$, 陈亚南 ${ }^{1}$ \\ ${ }^{1}$ 自然灾害研究所, 东北师范大学环境学院, 长春 130024 , 中国 \\ 2 资源与环境学院, 东北农业大学, 哈尔滨 150030 , 中国}

\begin{abstract}
Based on daily data recorded in 13 weather stations in Songnen grassland over the period 1982-2013, temporal variations in drought were investigated using the standardized precipitation evapotranspiration index (SPEI) and characteristics in NDVI were also analyzed using a time series data set of GIMMS NDVI3g during growing season (from April to October). In addition, for exploring the effects of climate change on vegetation, the correlation relationships between SPEI and NDVI were studied at different time scales. The results showed that the spatial distribution of NDVI presented a gradually reducing characteristic from the northeastern to southwestern. The NDVI showed an increasing trend with a slope of 0.0004 . Monthly NDVI exhibited the same pattern, with higher mainly ranged from July to September. The SPEI index experienced a downward trend and the drought often occurs in every month except in April. As a whole, this paper found that the correlation relationship between NDVI and SPEI was weak besides June and September. The lagging effect that existed in the impact on vegetation of drought was no significant. However, the lagging effect of one month lag was better than two month lag.
\end{abstract}

*通讯作者: 张继权, Zhangjq022@nenu.edu.cn
Keywords: NDVI; Drought; Standardized precipitation evapotranspiration index; Regional Response; Songnen grassland

\section{摘要}

基于松嫩草地 13 个气象站点的 1982-2013 年逐日气 象资料与牧草生长季 (4-10 月)逐旬 GIMMS NDVI3g 数据, 利用标准化降水指数 (SPEI) 分析了区域干旱 演变, 并与 NDVI 时间动态特征做相关分析, 进而探 讨气候变化对植被的影响。结果表明, 1982-2013 年 区域 NDVI 空间分布呈现由东北-中部-西南部逐渐降 低的特征; NDVI 时间变化呈波浪式缓慢上升趋势, 其趋势线斜率为 0.0004 ; 各月份变化大致相同, 波峰 区域基本集中在 7-9 月份; 32 年间, SPEI 指数体现 出区域干旱化加剧的趋势, 除 4 月外, 其他月份均有 不同程度的干旱发生。总体上, 除 6 月与 9 月外, 松 嫩草地 NDVI 与 SPEI 响应关系不强, 滞后效应不显 著, 但 1 个月滞后效应要好于两个月尺度的滞后响应 关系。

关键词: 标准化降水蒸散指数; NDVI; 干旱; 区域 响应; 松嫩草地

\section{1. 引言}

植被是地表主要的初级生产者之一, 位于大多数陆地 系统食物链的开端, 它也是土壤圈 -水圈-生物圈-大 
Risk Analysis and Crisis Response in Big Data Era (RAC-16)

气圈相互连接的纽带, 在全球碳平衡与大气稳定中发 挥着不可替代的作用。它不仅是区域气候特征的反映 和指示, 也受区域气候条件的制约 $[1,2]$, 二者之间 息息相关。因此, 自 20 世纪 90 年代以来, 植被与气候 变化的关系研究已成为气候变化研究领域关注的热 点。当前, 关于植被对气候变化响应研究中, 通常采 用归一化植被指数（NDVI，Normalized Difference Vegetation Index）监测植被的动态变化。该指数与植 被覆盖度、叶面积指数、植被生理条件等具有较好的 联系[3], 因此被直接用来表征植被的光合作用活性, 进而监测植被对气候变化的响应[4]。

干旱是世界上危害最为严重的自然灾害之一, 出 现次数多、影响范围广、造成危害大、持续时间长 [5]。 尽管干旱现象产生原因非常复杂, 但仍可以用相应的 干旱指数进行表征, 如标准化降水指数 (SPI)、Palmer 干旱指数 (PDSI)、农作物湿润指数 (SMI) 等[6]。 基于干旱指数可以用来量化干旱变化的强度、持续时 间以及发生的空间范围等 [7]。本研究中选取 Vicente-Serrano 于 2010 年提出的标准化降水蒸散指 数 [8] (SPEI, Standardized Precipitation Evapotranspiration Index) 分析干旱的动态变化。该指 数自提出以来, 被广泛应用至干旱分析的各领域, 因 为它不仅考虑了降水与蒸散在干旱成因中的作用, 而 且保留了 SPI、PDSI 等指数对于温度与降水的敏感 程度, 同时也具有多尺度与多空间比较的优点。干旱 频繁发生和长期持续阻碍了牧草正常返青和生长发 育, 造成草原生生产力明显下降, 对区域畜牧业经济 的可持续发展造成巨大威胁 [9]。因此, 监测并揭示 区域植被对干旱的响应关系, 在气候变化与陆地生态 系统关系的研究中具有重要的理论意义。
近年来, 已有学者对植被与干旱之间的响应关系 进行了相关研究。如王宏[6]等利用 NOAA/AVHRR NDVI 指数与 SPI 指数研究了中国北方荒漠草原、典 型草原、草甸草原与干旱气候的线性关系, 并基于虚 拟变量的回归模型模拟出二者之间的响应关系; 刘世 梁 ${ }^{[5]}$ 等基于逐旬 SPOT-NDVI 值与多尺度 SPEI 指数, 分析了云南省 1997-2012 年干旱演变与 NDVI 时间动 态变化特征; 李秀花 [1]等通过 NOAA/AVHRR NDVI 数据与同期气象资料, 借助遥感技术和数理统计知识, 分析了 1981-2001 年间中国西北干早区 NDVI 与气候 变化之间的线性关系。当前研究中主要研究 NDVI 与单一气候因子的响应变化, 并不能综合反映出气候 变化的影响[5]; 另一方面, 在 NDVI 与干旱响应分 析中, 研究大多数针对流域尺度进行分析, 且研究时 间跨度较短, 对牧区草地等关注较少, 尤其是有关松 嫩草地的研究未见报道。

本文以最新发布的 GIMMS NDVI3g 数据为基础, 基于 ArcGIS 和 ENVI 遥感数据处理平台, 对松嫩草 地 1982-2013 年间牧草生长季 (4-10 月) 植被覆盖情 况进行了动态监测, 同时基于 SPEI 指数分析了区域 干旱动态变化。此外, 本研究对松嫩草地 NDVI 与 SPEI 指数进行了不同尺度的相关分析, 进一步对区 域植被变化及其对气候变化的响应机制进行探究, 为 区域制定应对气候变化与防灾减灾对策及合理布局 水资源[10]提供了科学依据, 具有重要的理论和实际 意义。

\section{2. 研究区概况}

松嫩草原位于东北的中部 (图 1), 地处北纬 $43^{\circ} 30^{\prime}$ $48^{\circ} 05^{\prime}$ 、东经 $122^{\circ} 12^{\prime}-126^{\circ} 20^{\prime}$, 是我国三大平原之一

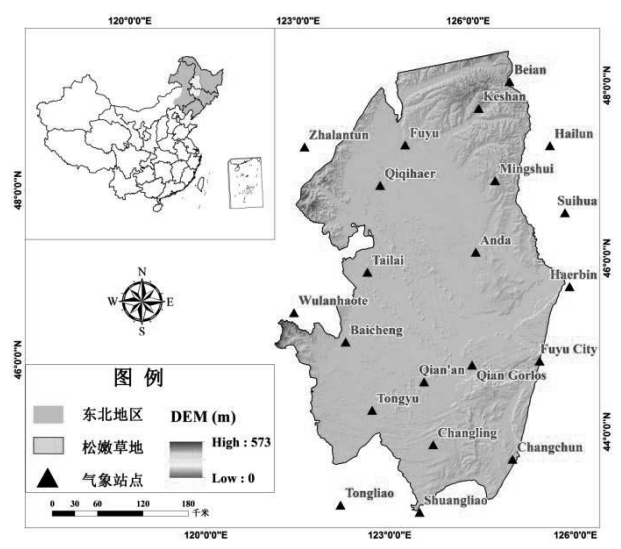

图 1. 研究区地理位置及气象站点分布图 
东北平原的主要组成部分。研究区天气变化比较频繁, 其气候特点是春季十年九旱, 干燥多风; 夏季潮湿温 热多雨; 秋季降雨适量、阳光充足、气候温和; 冬季 漫长寒冷少雪, 亦干燥多风。最暖月（7 月）平均气 温为 $22-25^{\circ} \mathrm{C}$, 最冷月 (1月)平均气温为 $-16--22^{\circ} \mathrm{C}$, 无霜期 $120-150 \mathrm{~d}$ 。春季在日平均温度达 $5^{\circ} \mathrm{C}$ 时, 多 年生牧草开始返青。年降水量为 $350-500 \mathrm{~mm}$, 干燥 度 1.1-1.5, 为草甸草原带。降水量自东向西递减, 年 蒸发量大于降水量的 2- 3 倍以上, 海拔多在 $150-250 \mathrm{~m}$ 。 研究区的土壤类型变化很大, 因此植被类型也多 样, 是欧亚草原带植被类型最丰富的地带。同时, 松 嫩平原也是我国重要的粮食主产区和商品粮基地。多 年来, 由于气候变化与人类活动的综合影响, 导致草 原产草量下降, 草畜矛盾日益尖锐, 严重阻碍了区域 草地畜牧业生产的发展[11]。

\section{3. 数据与方法}

\section{1. 数据来源与处理}

1) GIMMS NDVI 数据。本文所使用的遥感数据时间 序列为 1982-2013 年共 32 年, 本文选取每年的牧草 生长季, 即 4-10 月份作为研究时段。数据来源于 GIMMS 收集处理的 NOAA/AVHRR NDVI3g 第三代 全球植被数据集, 是空间分辨率为 $8 \mathrm{~km}$ 的 $15 \mathrm{~d}$ 最大 化合成产品, 共处理 448 幅影像。该数据集已经过大 气校正、辐射校正、几何校正、卫星轨道的漂移、云 量、太阳高度角等导致的气溶胶对数据质量的影响等 处理[12]。同时采用国际通用的最大合成法 MVC 获 取每月 NDVI 分布图, 采用均值法获得每年生长季 NDVI 平均值及 32 年间平均 NDVI 值分布图。

2) 气象数据。选用 1981-2013 年松嫩草地 13 个 地面气象观测站（图 1) 逐年的日降水量及温度的实 测资料, 对各气象台站数据进行了严格的质量审查, 审查发现各要素资料具有较好的连续性。数据来源于 中国气象数据共享服务网( http://cdc.cma.gov.cn/)。

\section{2. 研究方法}

1) SPEI 指数。SPEI 作为表征干旱一种多时间尺度 的指标，其计算过程同 SPI 相似，不同的是 SPI 仅考 虑了月降水量, 而 SPEI 同时考虑了月降水量和月潜 在蒸发量。具体计算主要有如下几个步骤[8]:

（1）计算潜在蒸散发PET，用 Thornthwaite 方 法求得。

（2）计算水分亏缺, 为月降水量与月潜在蒸散 之差, 公式为:

$$
D_{i}=P_{i}-P E T_{i}
$$

式 (1) 中, $D_{i}$ 为月水分亏缺量; $P_{i}$ 为月降水量; $P E T_{i}$ 为 月潜在蒸散发量, 单位均为 $\mathrm{mm}$ 。

（3）建立不同时间尺度气候学意义的水分盈亏 累积序列:

$$
D_{n}^{k}=\sum_{i=0}^{k-1}\left(P_{n-1}-P E T_{n-i}\right), n \geq k
$$

式中, $k$ 为时间尺度 (月); $n$ 为计算次数。

(4) 将 (3) 中所建序列采用 3 个参数 log-logistic 概率密度函数拟合:

$$
f(x)=\frac{\beta}{\alpha}\left(\frac{x-\gamma}{\alpha}\right)^{\beta-1}\left[1+\left(\frac{x-\gamma}{a}\right)\right]^{-2}
$$

式中: $f(x)$ 为概率密度函数, $\alpha 、 \beta$ 和 $\gamma$ 分别为尺度参 数、形状参数和 origin 参数, 可通过线性矩法进行估 算。累积概率函数为:

$$
F(x)=\left[1+\left(\frac{x-\gamma}{\alpha}\right)^{\beta}\right]^{-1}
$$

(5) 对序列进行标准正态分布转换, 得到相应 SPEI:

$$
S P E I=W-\frac{c_{0}+C_{1} W+C_{2} W^{2}}{1+d_{0} W+d_{2} W^{2}+d_{3} W^{3}}
$$

式中: $\mathrm{W}=\sqrt{-2 \ln (P)}$; 当 $\mathrm{P} \leq 0.5$ 时, $\mathrm{P}=1-F(x)$; 当 $\mathrm{P}>0.5$ 时, $\mathrm{P}=1-\mathrm{P}, \mathrm{SPEI}$ 的符号被逆转。常数 $C_{0}=2.515517, C_{1}=0.802853, C_{2}=0.010328$, $d_{1}=1.432788, d_{2}=0.189269, d_{3}=0.001308$ 。

2) 其他方法

将区域气象站点分布图与各 NDVI 时间序列叠 加, 选取距每个站点最近的 9 个像元作空间平均, 作 为与站点对应的 NDVI 值。利用一元线性回归分析 NDVI 时间序列变化趋势, 然后将各站点 NDVI 值序 列与 SPEI 值序列进行 Person 相关分析, 探究二者之 间的响应关系。

\section{4. 结果分析}

\subsection{NDVI 空间分布}

对松嫩草地内部 13 个气象站点 32 年来逐年牧草生长 季 NDVI 取平均值, 得到松嫩草地 NDVI 空间分布图。 由图 2 可知, 1982-2013 年, 松嫩草地 NDVI 生长季 平均值为 0.50 。存在一定的空间差异, 大部分的地 区的 NDVI 介于 $0.40-0.50$ 之间。整体而言区域 NDVI 北部高于南部, 西部高于东部, 呈现由东北-中部-西 南部逐渐降低的特征。

\subsection{NDVI 时间变化趋势}

1982-2013 年, 牧草生长季 NDVI 值变化曲线及其趋 势线见图 3。由图可知, 32 年间松嫩草地牧草生长季 NDVI 呈现波浪式缓慢发展趋势, 而趋势斜率为正, 


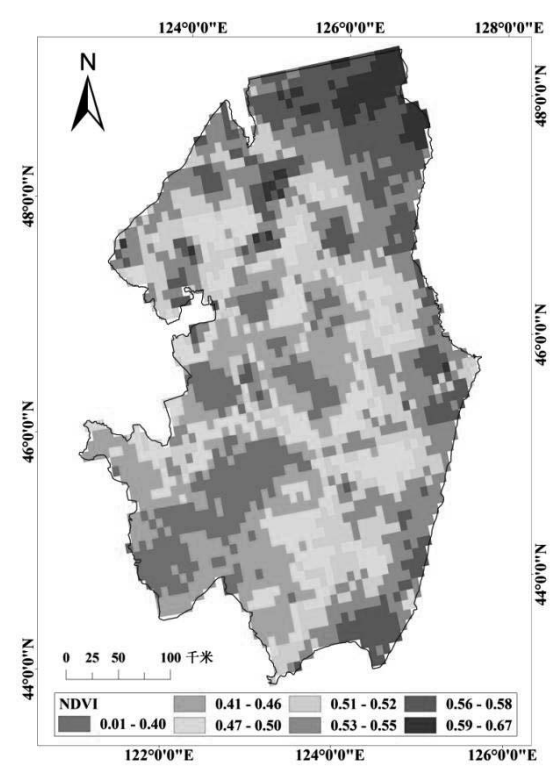

图 $21982-2012$ 年松嫩草地 NDVI 空间分布

说明 NDVI 总体略呈增加的态势。1990 年、1999 年、 2012 年和 2013 年 NDVI 值相对较大, 可能与当年丰 富的降雨量有关。生长季 NDVI 值均保持在 0.46 0.52 之间, 表明区域 NDVI 具有相对稳定性, 总体 植被状况较为乐观。

将 13 个站点的 NDVI 月最大值取平均值, 得到 松嫩草地月平均变化 NDVI 图（图 4)。如图所示, 可以看出松嫩草地生长季 NDVI 年内各月变化情况 大致相同,NDVI 变化波峰区域基本集中在 7 - 9 月份。 各年份之间波动情况相近, 具有周期性, 但周期内的 变化曲线有时也会出现波动[5]。最典型的是 1999 年

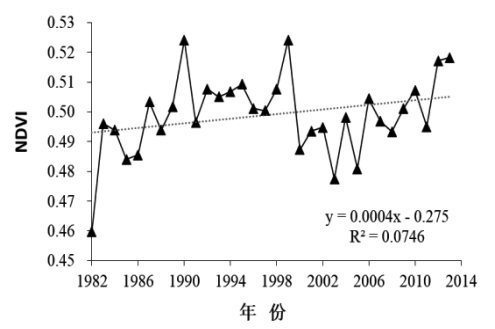

图 $31982-2013$ 年松嫩草地生长季 NDVI 变化
8 月, 比其他同一年份同时期的 NDVI 值偏大。从 NDVI 生长季各月份波动情况可以看出, NDVI 值与 气温及降雨量有一定的关系[13]。

\section{3. 松嫩草地 SPEI 指数的时间序列变化特征}

1982-2013 年松嫩草地 13 个气象站生长季 SPEI 的平 均值变化趋势见图 5。由图可知, 区域整体 SPEI 呈 现波动下降的趋势, 即区域整体干旱化趋势加强, 下 降幅度为 $-0.255 / 10 \mathrm{a}$ 。因为本研究计算的 SPEI 是基于 7 个月的时间尺度, 该尺度为中尺度, 受多雨期和少 雨期的影响, 数值波动较大, 但可反映出松嫩草地干 旱持续时间达到半年的干旱发生频率。因此, 根据图 5, 干旱持续时间半年且干旱较为严重的年份为 2000 年、 2001 年、 2004 年和 2007 年。

将 32 年间生长季内各月份 SPEI 值取月平均值进 行分析, 结果见图 6。可以看出, SPEI 的变化呈现先 降低再升高再降低的趋势。4 月份为牧草返青期, 干 旱程度较低, 有益于牧草返青与前期生长; 其次 7-8 月份为轻微干旱出现, 剩余月份干旱较为严重, 这与 全年降雨量分布大体一致。

\section{4. 松嫩草地 NDVI 动态变化与 SPEI 指数的关系}

利用松嫩草地各站点 $1982-2013$ 年生长季 SPEI 和 NDVI 数据, 分析不同年份二者之间的相关系数 $\mathrm{r}$, 探讨二者变化的相关性和滞后性。当 $r>0$ 时, 表示两 变量正相关, 当 $r<0$ 时, 表示两变量负相关, $r$ 的绝 对值大小代表相关程度。

表 1 给出各时间尺度下 NDVI 与 SPEI 值之间的 相关系数, 包括分析同一时间尺度下 NDVI 与 SPEI 值之间的相关系数, 以及 NDVI 分别滞后 1、2 个月 与 SPEI 之间的相关系数。从表 1 可以看出, 同一时 间尺度下 NDVI 与 SPEI 各站点的相关系数

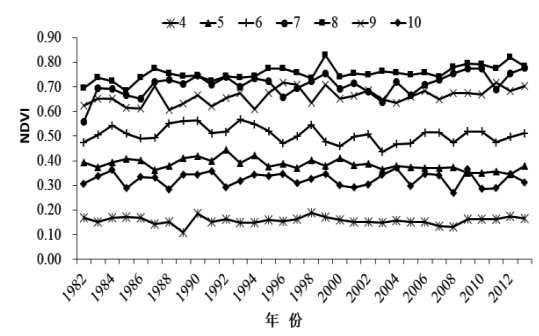

图 41982 - 2013 年松嫩草地生长季各月份 NDVI 变化 
Risk Analysis and Crisis Response in Big Data Era (RAC-16)

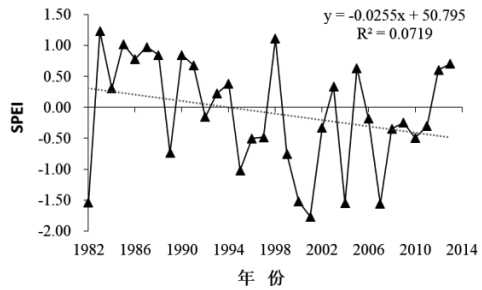

图 $51982-2013$ 年松嫩草地生长季平均 SPEI 变化

大多数均为负值, 6 月与 9 月份负相关系数最强; 仅 在 4、6 月份和整个生长季尺度呈现正相关, 相尺度 的 NDVI 与 SPEI 的相关系数, 多数为正相关, 相关 性不显著, 但滞时一个月 NVDI 与 SPEI 的正相关出

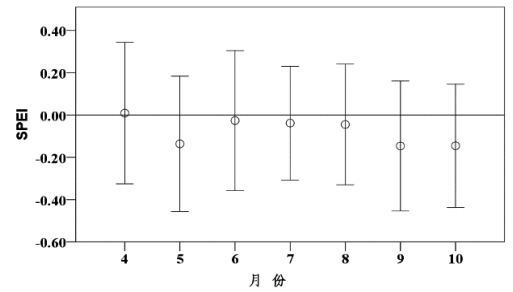

图 61982 - 2013 年松嫩草地生长季月 SPEI 变化

现月份较多。上述分析表明, 除了 6 月与 9 月份外, 区域 NVDI 对 SPEI 指数响应程度不强; 总体而言, 响应的滞后效应不明显, 但滞后一个月的响应关系要 优于滞后两个月尺度的响应关系。

表 1 松嫩草地 NDVI 与 SPEI 相关系数

\begin{tabular}{ccccccccccc}
\hline 时间 & & 4 & 5 & 6 & 7 & 8 & 9 & 10 & 生长季 \\
\hline \multirow{2}{*}{ NDVI } & $\max$ & 0.42 & 0.14 & 0.48 & 0.28 & 0.15 & 0.21 & 0.19 & 0.36 \\
& $\operatorname{mean}$ & 0.01 & -0.11 & 0.20 & -0.01 & -0.10 & -0.20 & -0.01 & 0.10 \\
\multirow{2}{*}{ 滞时一个月 NDVI } & $\max$ & 0.38 & 0.10 & 0.27 & 0.36 & 0.31 & 0.51 & 0.43 & - \\
& $\operatorname{mean}$ & 0.01 & -0.10 & 0.06 & 0.08 & 0.03 & 0.09 & 0.10 & - \\
滞时两个月 NDVI & $\max$ & 0.46 & 0.21 & 0.41 & 0.70 & 0.24 & 0.13 & 0.39 & - \\
& $\operatorname{mean}$ & 0.12 & -0.09 & 0.11 & 0.17 & -0.01 & -0.08 & 0.04 & - \\
\hline
\end{tabular}

注: $\max$ 为各时间尺度下 NDVI 与 SPEI 相关系数的最大值; mean 为各时间尺度下 NDVI 与 SPEI 相关系数的平均值; 表示未进行相关性分析。

\section{5. 讨论与结论}

\section{1. 讨论}

1）NDVI 变化趋势研究过程中发现 1990 年、1999 年、 2012 年和 2013 年 NDVI 值相对较大。经查证, 这几年降水均较为丰富, NDVI 值偏大可能与当年的 降水量充沛有关;

2) 本研究基于 SPEI 指数分析干旱化趋势, 研究 表明区域干旱化程度有加剧的趋势; 研究结果与郑盛 华[14]等人对松嫩平原 1961-2013 年干旱变化特征分 析的研究结论基本一致。

3) 在分析 NDVI 与 SPEI 相关性的过程中, 发现 二者之间相关性较弱, 仅在 6 月与 9 月较强, 这可能 是由于本研究选取的 NDVI 产品分辨率较大, 将 NDVI 提取至点然后进行相关分析, 会造成一些误差; 另一方面, 对于 SPEI 与 NDVI 呈现负相关的可能解 释是由于降水较大, 温度降低, 会导致植被落叶、枯 黄、脱落等, 从而减低 NDVI 值, 在二者响应关系上 被表征为负相关关系, 因此干旱会导致植被 NDVI
的增加。其他相关原因有待深入讨论。

\section{2. 结论}

本文基于 GIMMS NDVI3g 数据, 利用最大值合成法、 均值法、线性回归及相关分析法, 基于 ENVI 和 ArcGIS 软件平台, 对松嫩草地近 32 年植被覆盖时空 规律及其对干旱的响应特征进行分析。

本文研究结果表明, 1982- 2013 年间区域 NDVI 呈现由东北向西南部逐渐降低的特征; 时间变化上开 看, 区域植被覆盖整体变化不大, 呈缓慢波动上升的 趋势。 32 年来, SPEI 指数呈降低的趋势, 下降幅度 为 $-0.255 / 10 \mathrm{a}$, 表明区域干旱化趋势进一步加强。在 植被 NDVI 对干旱的响应上来看, 二者响应关系较强 的月份为 6 月和 9 月, 二者之间滞后效应不强。本文 研究结果可为区域制定防灾减灾, 生态恢复建设及水 资源合理优化布局提供科学的参考依据。

\section{Acknowledgement}

This study was financially supported by the National 


\section{Risk Analysis and Crisis Response in Big Data Era (RAC-16)}

Science and Technology Pillar Program during the 12th Five-Year Plan Period No.2013BAK05B02 and No. 2013BAK05B01.

\section{致谢}

本研究得到了“十二五”国家科技支撑项目 （2013BAK05B02）和（2013BAK05B01）的资助。

\section{6. 参考文献}

[1] 李秀花, 师庆东, 郭娟, 等. 中国西北干旱区 1981 2001年NDVI对气候变化的响应分析. 7 旱区资源与环境, 23(02): 12-16, 2009.

[2] Pennington D D, Collins S L. Response of an aridland ecosystem to interannual climate variability and prolonged drought. Landscape Ecology, 22(06): 897-910, 2007.

[3] 刘少华，严登华，史晓亮，等. 中国植被NDVI 与气候因子的年际变化及相关性研究. 干旱区 地理,37(03): 480-489, 2009.

[4] Zhang Y, Gao J, Liu L, et al. NDVI-based vegetation changes and their responses to climate change from 1982 to 2011: A case study in the Koshi River Basin in the middle Himalayas. Global and Planetary Change, 108: 139-148, 2013.

[5] 刘世梁, 田暳铎, 尹艺洁, 等. 云南省植被NDVI 的时间变化特征及其对干旱的响应. 生态学报, (15): 1-9, 2016.

[6] 王宏, 李晓兵, 李霞, 等. 中国北方草原对气候 干早的响应. 生态学报,28(01): 172-182, 2008.

[7] Mishra A K, Singh V P. A review of drought concepts. Journal of Hydrology, 391(1-2): 202-216, 2010.

[8] Vicente-Serrano S M, Beguería S, López-Moreno J I. A Multiscalar Drought Index Sensitive to Global Warming: The Standardized Precipitation Evapotranspiration Index. Journal of Climate, 23(7): 1696-1718, 2010.

[9] 马齐云, 张继权, 王永芳, 等. 内蒙古牧区牧草 生长季干旱特征及预测研究. 干旱区资源与环 境. 30(07): 157-163, 2016.

[10] Zhang R, Zou H, Hong M, et al. Risk Analysis of Water Resources Crisis in the Lancang-Mekong River Drainage Basin under the Background of Climate Change. Journal of Risk Analysis and Crisis Response, 2(3): 209-213, 2012.

[11] 柴风久, 罗新义, 李红. 松嫩草原现存问题与对 策. 黑龙江畜牧科技, (03): 43-44, 1998.

[12] Du J, Zhao C, Shu J, et al. Spatiotemporal changes of vegetation on the Tibetan Plateau and relationship to climatic variables during multiyear periods from 1982-2012. Environmental Earth Sciences, 75(1) ， 2016.doi 10.1007/s12665-015-4818-4

[13] Xu Y, Yang J, Chen Y. NDVI-based vegetation responses to climate change in an arid area of China. Theoretical and Applied Climatology, 2015.doi 10.1007/s00704-015-1572-1

[14] 郑盛华, 覃志豪, 张文博. 松嫩平原干旱变化特 征及其对气候变化的响应。中国农业气 象,36(05): 640-649, 2015. 